\title{
On the derivation of the Gibbs-Helmholtz equation
}

\author{
Balázs Roósz $^{1} \cdot$ Csaba Visy $^{1} \cdot$ István Nagypál $^{1}$
}

Received: 1 February 2016/Accepted: 19 February 2016/Published online: 4 April 2016

(c) Springer International Publishing Switzerland 2016

\begin{abstract}
Based on a real event in the classroom during the physical chemistry course for undergraduate students, a new derivation is presented for the proof of the GibbsHelmholtz equation. Tried in subsequent years, it received positive reflection from chemistry BSc students proving that this straightforward derivation, which leads from the definition of $G$ to the final expression in five steps, is more easily comprehensible. At the same time it helps the instructor to shed light-based on their temperature dependence-on the relationship between thermodynamic state functions.
\end{abstract}

Keywords Physical chemistry - Thermodynamics .

Undergraduate students $\cdot$ More comprehensible derivation

\section{Introduction}

There are several paths in the different textbooks [1-5] for the derivation of the Gibbs-Helmholtz (G-H) equation:

$\left[\frac{\partial G / T}{\partial T}\right]_{p}=\frac{-H}{T^{2}}$

The Gibbs-Helmholtz equation expresses the temperature dependence of the ratio of $G / T$ at constant pressure, which is a composite function of $T$ as $G$ itself also depends on the temperature.

Csaba Visy

visy@chem.u-szeged.hu

1 Department of Physical Chemistry and Materials Science, University of Szeged, Rerrich sq. 1, Szeged 6720, Hungary
This fundamental equation is very important, since it is the starting point to the van't Hoff equation, expressing the temperature dependence of the equilibrium constant that interprets quantitatively the shift of chemical equilibrium upon temperature change, predicted in the Le ChatelierBrown principle.

In a widely used physical chemistry lecture book [1] the starting point to the $\mathrm{G}-\mathrm{H}$ equation is the definition of Gibbs' free energy, and the relation between its temperature coefficient and entropy:

$G=H-T S ;-S=\left(\frac{\partial G}{\partial T}\right)_{p}$.

As a next step, these two equations are combined to result

$\left(\frac{\partial G}{\partial T}\right)_{p}=\frac{G-H}{T}$

and a transformation gives

$\left(\frac{\partial G}{\partial T}\right)_{p}-\frac{G}{T}=-\frac{H}{T}$.

Then it is suggested to recognize the validity of the equation

$\left(\frac{\partial G}{\partial T}\right)_{p}-\frac{G}{T}=T\left(\frac{\partial}{\partial T} \frac{G}{T}\right)_{p}$

However, this conclusion is not self-evident for the average chemistry student, as its approval needs not only the derivation of a composite function:

$$
\left(\frac{\partial}{\partial T} \frac{G}{T}\right)_{p}=\frac{1}{T}\left(\frac{\partial G}{\partial T}\right)_{p}+G\left(\frac{\partial}{\partial T} \frac{1}{T}\right)_{p}
$$

but also a comparison with the rearranged form of Eq. 4: 
$\left(\frac{\partial}{\partial T} \frac{G}{T}\right)_{p}=\frac{1}{T}\left[\left(\frac{\partial G}{\partial T}\right)_{p}-\frac{G}{T}\right]=\frac{-H}{T^{2}}$

As it can be seen, the train of thought is not totally straightforward, which hampers the logic of the derivation. According to classroom experiences, the derivation rises difficulties in the sense that it is not so easy to realize that the left side of Eq. 5 is the product of $T$ with the derivative of $G / T$.

Some years ago during the Physical Chemistry course, one of the then students (B.R.) of us (C.V. and I.N.) suggested the following derivation: let us take the definition of G

$G=H-T S$

and divide both sides by $T$

$\frac{G}{T}=\frac{H}{T}-S$

If we derive this equation with respect to $T$ at constant pressure,

$\left[\frac{\partial\left(\frac{H}{T}-S\right)}{\partial T}\right]_{p}=\frac{1}{T}\left(\frac{\partial H}{\partial T}\right)_{p}-\frac{H}{T^{2}}-\left(\frac{\partial S}{\partial T}\right)_{p}$

we receive Eq. 10 consisting of three terms. Comparing it with Eq. 1, it can be realized that the first and the third terms are missing, they assumingly have cancelled each other. Seemingly, it might be also the result if $H$ and $S$ were independent of the temperature. Since it is evidently not the case recognized also by himself, this striking coincidence prompted us to find an easily comprehensible proof, and to show the students through a simple reasoning, why the sum of these two terms in Eq. 10 gives zero.

For the first term we have

$\frac{1}{T}\left(\frac{\partial H}{\partial T}\right)_{p}=\frac{C_{p}}{T}$

In order to receive the third term, we consider that for the infinitesimal change in entropy at constant pressure we have

$d S=\frac{d q_{\mathrm{rev}}}{T}=\frac{C_{p} d T}{T}$

Equation 12 implies that

$\left(\frac{\partial S}{\partial T}\right)_{p}=\frac{C_{p}}{T}$

so that we obtained the same expression for the first and third element in Eq. 10. Since the first and third term at the right side in Eq. 10 are identical but with opposite sign, they are cancelled. Quod erat demonstrandum.
Thus, Eqs. 8-13 represent a direct, straightforward path to obtain $\mathrm{G}-\mathrm{H}$ equation from the definition of $G$ in five steps. We are aware that in one of the referred textbooks [4] the derivation through Eqs. (8-10) and (13) is presented. However, McQuarrie and Simon have to refer also to previous equations to prove validity of Eq. 13, where they come to the conclusion in four further steps. Thus, the derivation consists altogether of eight elements. We emphasize that our derivation in five steps includes the concrete proof that the sum of the first and third term in Eq. (10) is zero.

It is illuminating to point out that-assuming that $H$ and $S$ were temperature independent quantities-we could derive the Gibbs-Helmholtz equation in one step from Eq. 10. But the assumption is evidently wrong. The coincidence, however, reveals that the first derivatives of $H$ and $S$ with respect to temperature are related, and this relation results in the elimination of the first and third terms in Eq. 10 .

Finally we may mention in the basic course the so-called compensation effect [6]. It expresses that the change of $G$ is, in general, much less sensitive to the change of the different parameters than that of the change of $H$ and $S$. This is clearly reflected in the above mentioned elimination when the changing parameter is the temperature. Further details of the compensation effect, however, are treated in the master courses.

Although we do not have at hand exact statistical data concerning the usage of physical chemistry lecture books, the presented straightforward derivation in this simple, tutorial form, leading from the definition equation of $G$ to the final form of the $\mathrm{G}-\mathrm{H}$ equation in five steps, is not found in the generally used textbooks [1-5]. Our experiences in the classroom are unambiguous that-when presented to our students during the subsequent semesters-majority of them reflected that it was easier to comprehend than the derivation given in their textbook [1]. Beyond the didactic advantage, the presented derivation and its arguments shed light on the fact that the temperature dependence of $H$ and $S$ are related, and that this relation is the basis of the compensation effect.

\section{References}

1. Atkins PW (2005) Physical chemistry, 6th edn. vol 1. Oxford Press, London, pp 45-46

2. Alberty RA, Silbey RJ (1913) Physical chemistry, 1st edn. vol 1. Wiley, New York (and its later editions), pp 117-118 
3. Noggle JH (1989) Physical chemistry, 2nd edn. vol 1. Scott, Foresman and Company, Glenview, pp 145

4. McQuarrie DA, Simon JD (1997) Physical chemistry: a molecular approach, vol 1. University Science Books, Sousalito, pp 854-855 and 902-903
5. Tester JW, Modell M (1997) Thermodynamics and its applications, 3rd edn. Prentice Hall, New Jersey, pp 153, 341

6. Benson SW (1976) Thermochemical kinetics, 2nd edn. Wiley, New York, pp 21-23 